\title{
TUY 40 Telescope Control System Based on LabVIEW
}

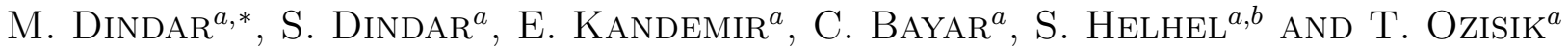 \\ ${ }^{a}$ TÜBİTAK National Observatory, R\&D Department, Antalya, Turkey \\ ${ }^{b}$ Akdeniz University, Engineering Faculty, EEE, Antalya, Turkey
}

\begin{abstract}
Events and transients are becoming more and more crucial in modern astronomy such as supernovae, gammaray bursts and so on. VOEvent messages have all information to make the follow-up observations for transients and events which forces the scientists to make robotic observations. Robotic autonomous telescopes usually run under the control of a scheduler, which provides high-level control by selecting astronomical targets for observation. TUY 40 is a new robotic autonomous telescope control system designed by TÜBİTAK National Observatory (TUG). TUY 40 is based on 16 inch Meade LX-200 telescope mount and optical tube without any Meade electronic components and drive motors. The telescope control software developed on open-source Talon software and LabVIEW is presented. The algorithm for telescope control (slewing, tracking, homing etc.) was implemented in National Instruments PXI chassis written in LabVIEW real-time software from scratch. Talon is open-source software based on the GNU/C UNIX platform. A new TCP/IP library was implemented within Talon software to communicate with hard real-time telescope control software in LabVIEW PXI chassis. Initial setup without any permanent pier and polar alignment showed that the pointing error of the telescope has been obtained as $2.22 \operatorname{arcmin}(132 \operatorname{arcsec})$ in RA axis (horizontal axis of the image) and 25 arcsec in Dec axis (vertical axis of the image), and tracking error has been observed as $\approx 4.8$ arcsec per second.
\end{abstract}

DOI: 10.12693/APhysPolA.128.B-73

PACS: 95.55.Cs, 87.85.St

\section{Introduction}

Design concept of the robotic telescope system has been developed and implemented over the last two decades. One of the earliest microcomputers based on telescope control system was introduced by the Indiana University group followed by investigator [1]. The advantage of the computer-based robotic telescope system was discussed elsewhere for low operating costs with high operating efficiency and high scientific productivity $[2,3]$.

Generally, the term robotic telescope stands for any telescope using advanced information technologies a computer is at least able to guide a telescope to given position and take images or is capable of more complicated tasks. The term autonomous observatory means a robotic telescope and dome is computer controlled in such manner, that all indispensable actions of observation are done automatically, including processing of weather conditions, dome driving, choosing objects to observe, exposing by cameras or other optical sensors, taking calibration images, and so forth [4].

This paper presents a new designed telescope control software (TCS), TalonVIEW, and adapted to observatory control software, Talon, in order to control the Meade LX-200 telescope mount as autonomous robotic telescope. This new TCS is called as TUY 40 Telescope Control Software.

\section{The architecture of TUY 40 robotic telescope \\ 2.1. Hardware architecture}

Motion control of the two main axes, RA and Dec, is implemented within motor drivers and motion

*corresponding author; e-mail: murat.dindar@tubitak.gov.tr controller cards. Two servo motors are used to move the axes. Precise motion control is a prerequisite for a telescope. Therefore, closed loop PID control is applied for accurate position control within high resolution motor drivers with encoders. Motion controller cards reliably execute programs with very precise timing. They are playing the key role to deliver the target position and velocity values sent by the software to the drivers.

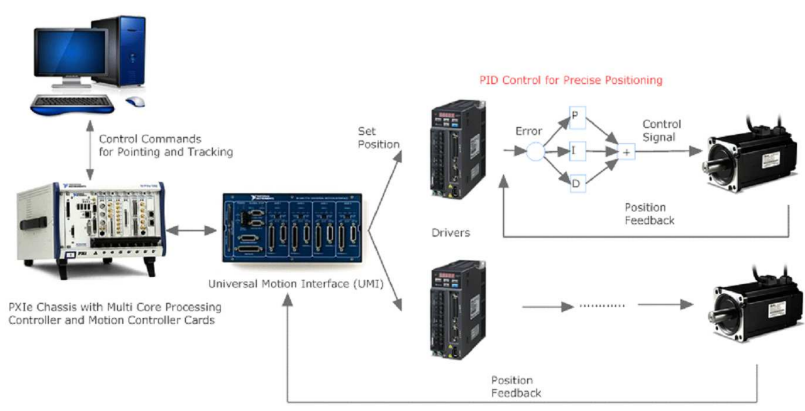

Fig. 1. Hardware architecture of TUY 40 telescope.

The commands coming from the computer is evaluated PXIe real-time embedded controller based on the codes written within LabVIEW and embedded in the controller. Then it sends appropriate values to the motion controller cards. Required pulse and direction voltages are sent to the drivers via universal motion interface (UMI) cards mounted in the PXIe chassis (Fig. 1). Servo driver receives the target position value and starts to move the motor. It reads encoder feedback signals and runs the PID control loop to carry out precise positioning within specified time interval.

\subsection{Software architecture}

The new TCS control software has two major parts, LabVIEW and Talon. Talon is open source observatory 
control software and written in GNU/C. It is used to make astronomical calculations, control camera and observatory modules such as GPS, meteo station, etc. [5]. Talon have already been used in T60 autonomous telescope in TUG in different software and hardware architecture. The new low-level TCS control system, TalonVIEW, is implemented on LabVIEW platform because of its rapid prototyping, debug features and IDE and integrated with Talon to develop the TUY 40 TCS.

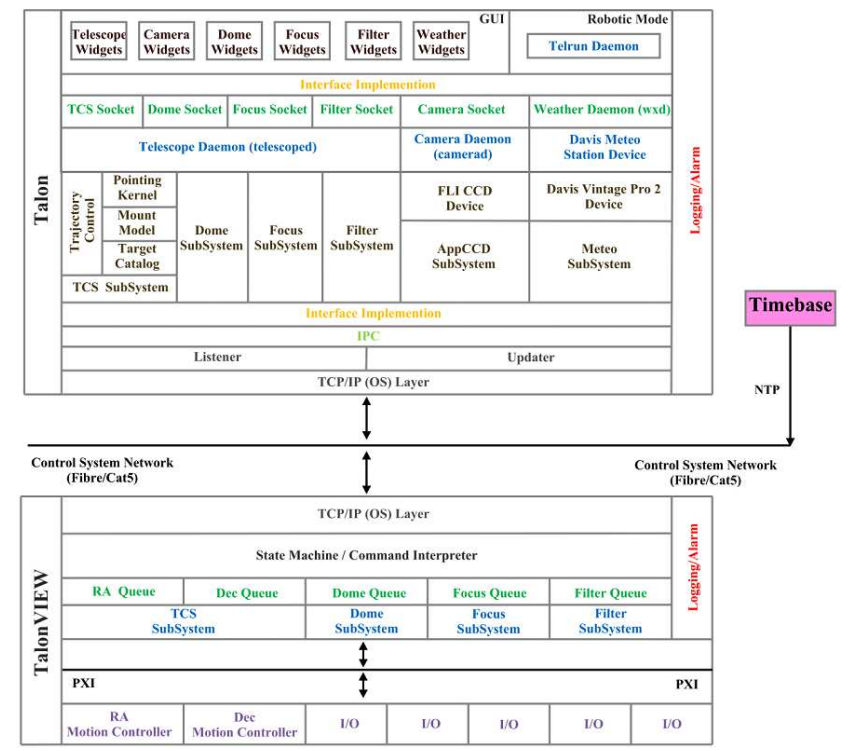

Fig. 2. The Software architecture of TUY 40 TCS.

The new architecture is simply based on the client/server model. The client side of new TCS software is based on Talon running on Linux, with a new TCP/IP library and application functions of this library. The server side of new TCS software is based on LabVIEW which is running on the PXI chassis with realtime embedded controller and motion controller cards. Several VIs and codes are written in LabVIEW and Talon from scratch to implement the architecture shown in Fig. 2.

\section{Test results}

In the test results of the telescope using very rough polar alignment without permanent pier, Canon EOS 20Da Digital SLR Camera was used as image detector system on the telescope focal plane. The camera has $3504 \times 2336$ pixel area. The pointing error of the telescope is 2.22 arcmin (132 arcsec) in RA axis and 25 arcsec in Dec axis as shown in Fig. 3. Tracking test of the telescope was made as well. For the tracking tests, three types of images were taken, 20, 60, and $100 \mathrm{~s}$. As a result of analyzing of those images, tracking error was found as 4.8 arcsec per second approximately.

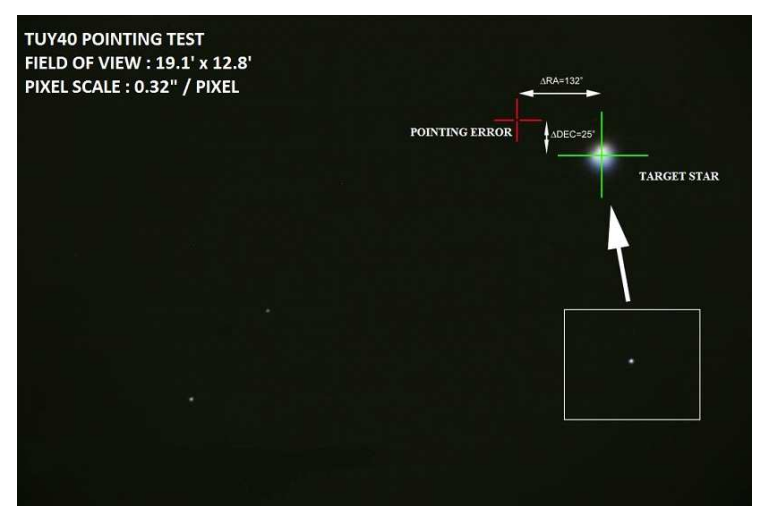

Fig. 3. Test image of TUY 40 telescope for pointing.

\section{Conclusion}

In this study, a new robotic TUY 40 TCS has been designed within Real-Time LabVIEW and implemented in NI-PXI Platform by integrating Talon, observatory control software. LX-200 Meade telescope mount was adapted to the new robotic telescope structure by using the new TCS software. The satisfying results were taken for pointing and tracking in the test stage. For upcoming study, telescope will be installed on a robust platform so that robust pointing model of the telescope will be generated and it makes the pointing and tracking errors smaller.

\section{Acknowledgments}

This project was granted by State Planning Organization (Grant number: 2010K120170-DPT), and TÜBİTAK National Observatory.

\section{References}

[1] R.K. Honeycutt, J.E. Kephart, A.A. Hendon, Sky Telescope 56, 495 (1978).

[2] L.J. Boyd, R.M. Genet, D.S. Hall, Sky Telescope 70, 16 (1985).

[3] M. Drummond, J. Bresina, W. Edgington, K. Swanson, G. Henry, E. Drascher, Flexible scheduling of automatic telescopes over the internet, Publications of the Astronomical Society of the Pacific, Vol. 79, 1995, p. 101.

[4] M. Nekola, R. Hudec, M. Jelínek, P. Kubánek, J. Štrobl, C. Polášek, Adv. Astron. 2010, 1-5 (2010).

[5] Reference Manual for OCAAS (Talon), Version 2.0, 2000 . 